\title{
Document Version Date
}

National Cancer Institute

\section{Source}

National Cancer Institute. Document Version Date. NCI Thesaurus. Code C93813.

The date (and time) on which the document is versioned. 